This is the Authors' Accepted Manuscript version of an article published in Statistics in Medicine in 2019, vol. 38, pages 778-791; DOI: 10.1002/sim.8010

\title{
Estimating multilevel regional variation in excess mortality of cancer patients using integrated nested Laplace approxima- tion
}

\author{
Karri Seppä ${ }^{1 *}$, Håvard Rue ${ }^{2}$, Timo Hakulinen ${ }^{1}$, Esa Läärä ${ }^{3}$, Mikko J. Sillanpää ${ }^{3,4}$, \\ Janne Pitkäniemi ${ }^{1,5}$ \\ ${ }^{1}$ Finnish Cancer Registry, Institute for Statistical and Epidemiological Cancer Re- \\ search, Helsinki, Finland \\ ${ }^{2}$ Department of Mathematical Sciences, Norwegian University of Science and Tech- \\ nology, Trondheim, Norway \\ ${ }^{3}$ Research unit of Mathematical sciences, University of Oulu, Finland \\ ${ }^{4}$ Biocenter Oulu, Finland \\ ${ }^{5}$ Department of Public Health, University of Helsinki, Finland
}

Correspondence: *Karri Seppä, Finnish Cancer Registry, Unioninkatu 22, FI-00130

Helsinki, Finland; karri.seppa@cancer.fi

\begin{abstract}
Models of excess mortality with random effects were used to estimate regional variation in relative or net survival of cancer patients. Statistical inference for these models based on the Markov chain Monte Carlo (MCMC) methods is computationally intensive and, therefore, not feasible for routine analyses of cancer register data. This study assessed the performance of the integrated nested Laplace approximation (INLA) in monitoring regional variation in cancer survival. Poisson regression model of excess mortality including both spatially correlated and unstructured random effects was fitted to the data of patients diagnosed with ovarian and breast cancer in Finland during 1955-2014 with follow-up from 1960 through 2014 by using the period approach with 5 -year calendar time windows. We estimated standard deviations associated with variation i) between hospital districts and ii) between municipalities within hospital districts. Posterior estimates based on the INLA approach were compared to those based on the MCMC simulation. The estimates of the variation parameters were similar between the two approaches. Variation within hospital districts dominated in the total variation between municipalities. In 2000-2014, the proportion of the average variation within hospital districts was $68 \%$ (95\% posterior interval: $35-93 \%)$ and $82 \%(60-98 \%)$ out of the total variation in ovarian and breast cancer, respectively. In the estimation of regional variation, the INLA approach was accurate, fast and easy to implement by using the R-INLA package.
\end{abstract}

Key words. cancer survival, excess mortality, integrated nested Laplace approximation (INLA), Markov chain Monte Carlo (MCMC), regional variation 


\section{Introduction}

Excess mortality of a patient diagnosed with a given disease is the additional hazard of death encountered by the patient compared to the hazard of death in a relevant reference population group. Monitoring excess mortality by region is important, because variation in the excess mortality may reflect regional differences in the effectiveness of cancer care.[1] A comprehensive national health service system should ensure the same level of cancer care to all people in the country.

In Finland, inhabitants of a given municipality are most often treated in their own central hospital and further referred to the pertinent university hospital, if more advanced hospital care is needed. Therefore, we are primarily interested in assessing regional variation across these central hospital districts or university hospital regions. However, equity across these hospital districts does not necessarily imply equity across municipalities within the districts.

The excess mortality can be summarised in terms of relative or net survival these quantities being often used in population-based cancer survival analyses to measure cancer survival after accounting for differences in reference population mortality.[2, 3, 4] They have been used e.g. in the international comparisons of cancer survival between countries, $[5,6]$ between regions within a country $[7,8]$ or between other population subgroups.[9] The same methods apply to the assesments of mortality associated with diseases other than cancer, too, e.g. cardiovascular [10, 11] and cerebrovascular diseases [12] and HIV.[13]

Excess mortality is often modelled by splitting follow-up time in pre-specified intervals within which binomial distribution for the number of deaths [14] or a piecewise constant excess hazard [2] is assumed. These models can be fitted in the framework of generalised linear models by using binomial or Poisson distribution with non-standard link-functions. [15] When a piecewise constant excess hazard is assumed, there is a good argument for using shorter intervals early in the follow-up, as patients' excess mortality often changes most rapidly in the beginning of follow-up.[15] Alternatively, baseline excess mortality has been parametrised by using mixture cure models [16] and flexible parametric models.[10] Modelling excess mortality with a non-parametric baseline excess hazard function has also been proposed.[17, 18]

The piecewise constant excess hazard model [15] was extended by Kuss et al[19] to account for clustered responses using spatially unstructured random effects. This model was fitted within the class of generalised linear mixed models by using maximum likelihood methods. In a Bayesian framework, a similar type of model with spatially correlated random effects has been used to assessing spatial variation in cancer survival by Fairley et al,[20] Cramb et al,[21] Saez et al[22] and Kang et al.[23] Recently, Cramb et al[24] extended a flexible parametric model [10] to estimation of spatial variation in excess mortality. The disadvantage of these Bayesian approaches is that they are computationally intensive,[24] because the models are fitted by using Markov chain Monte Carlo (MCMC) methods. 
In Bayesian inference, the integrated nested Laplace approximation (INLA) approach proposed by Rue et al[25, 26, 27] is computationally more efficient than the MCMC methods. The INLA approach can be easily applied by using the R-INLA package [25] (www.r-inla.org) to a wide variety of statistical models including generalised linear mixed models. In the estimation of excess mortality and net survival, the INLA approach has been mentioned as a possible solution to overcome the computational challenges related to the MCMC approaches, [24, 23] but to our knowledge, it has not been employed for the estimation in practice.

In this paper, we apply the piecewise constant excess hazard model with spatially correlated random effects to the follow-up data of patients diagnosed with the cancers of ovary and breast in Finland. Variation in cancer survival is assessed in two levels: across hospital districts and across municipalities within hospital districts. The model is implemented in a Bayesian framework using the INLA approach. The results are compared to those estimated by using the MCMC methods in order to validate the use of the INLA approach in the assessment of regional variation in excess mortality and net survival.

\section{Excess hazard model with regional random ef- fects}

\subsection{Patients diagnosed with ovarian and breast cancer}

We analysed the follow-up data of women diagnosed with the cancers of ovary and breast (primary invasive malignancies with ICD-10 codes C56 and C50, respectively) in Finland and reported to the Finnish Cancer Registry. The patients were diagnosed in 1955-2014 and followed up for death in 19602014. In Finland, specialised hospital care is currently provided by 20 central hospital districts.[28] In addition, the autonomous region of Aland Islands was considered as a separate hospital district in the analysis. Each central hospital district consists of member municipalities and is subsumed under one of the five larger cancer control regions, each being lead by a university hospital. The analyses were based on the regional division of 313 municipalities that existed in Finland in 2016 (Supplementary Figure S1). Patient's municipality was based on her place of residence at the beginning of the year of diagnosis.

The period approach [29] was used in the analysis. The follow-up data were split into 11 five-year period windows of calendar time: 1960-1964, 1965$1969, \ldots, 2010-2014$. For example, in period window 2000-2004, follow-up data in the first 5-year period after diagnosis are from patients diagnosed in 1995-2004 and followed-up in 2000-2004. Each patient contributed to one or two period windows, because follow-up times were censored at 5 years after diagnosis. Table 1 shows the number of patients diagnosed in each period window and the number of patients diagnosed in the preceding period, who contributed to the 5-year survival in the given period window with 
a late entry. All models considered were fitted separately for the 11 periods. Patient's age at diagnosis was categorised into 5 groups: 0-44, 45-54, 55-64, 65-74 years, and 75 years and older. With the exception that we included children aged $<15$ years, these age groups are nowadays often used, for example, in the CONCORD-3 [6] (this study excluding patients 100 years or older, though) and the EUROCARE-5 [5] studies. This age grouping is also the default selection in the SEER*Stat software [30] provided by the Surveillance, Epidemiology, and End Results (SEER) program, and used by Corazziari et al,[31] too, who proposed the International Cancer Survival Standard.

\subsection{Piecewise constant excess hazard model}

Let $h(t)$ be the hazard of death for a patient alive at time $t$ after diagnosis. The excess hazard of death $h_{\mathrm{E}}(t)=h(t)-h_{\mathrm{P}}(t)$ is the excess rate of death that the patient has compared to the rate of death $h_{\mathrm{P}}(t)$ in a comparable person in the reference population. The expected hazard of death for a patient $i$ in follow-up interval $j$ was determined by central hospital district $r_{i}(r=1, \ldots, 21)$, calendar year $k_{i j}(k=1960, \ldots, 2014)$ and age $a_{i j}$ in years $(a=0, \ldots, 99)$, i.e. $h_{\mathrm{P}}(t)=\lambda_{i j}=\lambda_{r_{i}} k_{i j} a_{i j}$

The excess hazard $h_{\mathrm{E}}(t)$ of death due to cancer was assumed to be constant within $J$ pre-specified follow-up intervals, i.e., $h_{\mathrm{E}}(t)=\nu_{j}$, when $t_{j-1} \leq t<t_{j}$ for $j=1,2, \ldots, J$. The excess hazard $\nu_{i j}$ of patient $i$ is modelled as a multiplicative function of covariates $\boldsymbol{x}_{i j}=\left(x_{i j 1}, \ldots, x_{i j b}\right)^{\prime}$, such that $\nu_{i j}=$ $\exp \left(\boldsymbol{\alpha}^{\prime} \boldsymbol{x}_{i j}\right)$ where $\boldsymbol{\alpha}=\left(\alpha_{1}, \ldots, \alpha_{b}\right)^{\prime}$ is the vector of regression coefficients, in which each $\alpha_{l}, l=1, \ldots, b$, is interpreted as the additive effect of the $l$ th covariate on the logarithm of the excess hazard.

The baseline model included the age group and follow-up time $(0-<4$ months, $4-<12$ months, and four annual intervals from 1 to 5 years) as categorical covariates. Interaction terms between the five age groups and three followup time intervals $(0-<4$ months, $4-<12$ months, and $1-<5$ years $)$ were also included to allow non-proportional excess hazards by age at diagnosis. The first five years of follow-up after diagnosis were considered and longer survival times were censored at five years.

The model was fitted within the framework of the generalised linear models by assuming a Poisson error for the observed number of deaths $d_{i j} \sim$ Poisson $\left[\left(\lambda_{i j}+\nu_{i j}\right) y_{i j}\right]$ and specifying link function $\ln \left(\mu_{i j}-\lambda_{i j} y_{i j}\right)$, where $y_{i j}$ is time at risk in interval $j$ for patient $i$ and $\mu_{i j}$ is the expectation of the Poisson distribution.[15] The Poisson model was fitted to collapsed data $\boldsymbol{Y}_{m g j}=\left(d_{m g j}, d_{m g j}^{*}, y_{m g j}\right)$ where the observed number $d_{i j}$ and expected number $d_{i j}^{*}=\lambda_{i j} y_{i j}$ of deaths and time at risk $y_{i j}$ were summed over patients in each stratum of region $m$ (hospital district or municipality), age group $g$, and follow-up time interval $j$. 


\subsection{Regional effects in excess mortality and net survival}

The excess hazard of death $\nu_{i j}$ may vary by hospital district $(r=1, \ldots, R)$ and by municipality $(m=1, \ldots, M)$ as follows:

$$
\nu_{i j}=\exp \left(\boldsymbol{\alpha}^{\prime} \boldsymbol{x}_{i j}+\beta_{r_{i}}+u_{m_{i}}+v_{m_{i}}\right),
$$

where $\beta_{r}$ is a hospital district specific effect, and $u_{m}$ is a structured and $v_{m}$ is an unstructured municipality-specific random effect. In our application $R=21$ and $M=313$.

The parameter vectors of random effects $\boldsymbol{\beta}, \boldsymbol{u}$ and $\boldsymbol{v}$ were assumed to be mutually independent. Hospital district specific effects $\beta_{r}$ were assumed to follow the normal distribution with mean zero and standard deviation $\sigma_{\beta}$. Unstructured heterogeneity across municipalities was modelled by multivariate normally distributed random effects $v_{m}$ with mean vector of zeros and covariance as $\sigma_{v}^{2} I$. Spatial dependencies between the municipalities were described by assuming intrinsic Gaussian conditional autoregression (CAR) [32] for the multivariate distribution of random effects $\boldsymbol{u}=\left(u_{1}, \ldots, u_{M}\right)$ :

$$
\left[u_{m} \mid \boldsymbol{u}_{-m}, \sigma_{u}\right] \sim \mathrm{N}\left(\frac{1}{n_{m}} \sum_{j \in N_{m}} u_{j}, \frac{\sigma_{u}^{2}}{n_{m}}\right),
$$

where $\boldsymbol{u}_{-m}$ denotes all components of $\boldsymbol{u}$ except $u_{m}, \sigma_{u}$ and $\sigma_{v}$ are the standard deviation parameters and $N_{m}$ is the set of neighbours of municipality $m$ and $n_{m}$ is the number of its neighbours. In the CAR model, if two municipalities are defined to be neighbours their random effects are correlated, while random effects in non-neighbouring municipalities are modelled as being conditionally independent given the remaining random effects.[33, 34] In our application, adjacent municipalities within the same hospital district are defined as neighbours, and within each hospital district, a sum-to-zero constraint is specified on the municipality-specific random effects.

The excess mortality can be summarised in terms of the net survival index that could be interpreted, under strong assumptions, as the survival probability in a hypothetical situation where the disease would be the only possible cause of death with all other causes being eliminated.[3, 4] Although the probability interpretation may not apply, net survival is an informative and widely-used measure for cancer survival after elimination of the influence of other causes of death. The age-standardised net survival [35] in municipality $m$ is the weighted average of age-specific net survival indices, i.e.,

$$
S_{\mathrm{E}}(t, r, m)=\sum_{g=1}^{G} w_{g} \exp \left[-\int_{0}^{t} \nu(g, u, r, m) d u\right],
$$

where the excess hazard is $\nu(g, t, r, m)=\exp \left[\boldsymbol{\alpha}^{\prime} \boldsymbol{x}(g, t)+\beta_{r}+u_{m}+v_{m}\right]$ and $w_{g}$ is the proportion of patients in age group $g(g=1, \ldots, G)$ at the beginning of follow-up. The excess mortality $\nu$ and net survival $S_{\mathrm{E}}$ were estimated separately in each 5-year calendar period and standardised to the average distribution of patients' age at diagnosis, i.e., $w_{g}$ was the proportion of patients diagnosed in Finland during the whole period 1960-2014 in age group $g$. 


\subsection{Quantifying regional variation}

The amount of regional variation in each 5-year calendar period was summarised by estimating i) variation between hospital districts, ii) variation between municipalities within hospital districts, and iii) total variation between municipalities. The variations were quantified both in the relative excess mortality and in the 5-year net survival.

Variation in the relative excess mortality was quantified on the logarithmic scale by the standard deviation $s_{\mathrm{B}}$ of the effects $\beta_{r}$ of $R$ hospital districts and the standard deviation $s_{\mathrm{W}}$ of the effects $u_{m}+v_{m}$ of $M$ municipalities, respectively, i.e.:

$$
s_{\mathrm{B}}^{2}=\frac{1}{R-1} \sum_{r=1}^{R}\left(\beta_{r}-\bar{\beta}\right)^{2} \quad \text { and } \quad s_{\mathrm{W}}^{2}=\frac{1}{M-1} \sum_{m=1}^{M}\left(v_{m}+u_{m}-\bar{u}-\bar{v}\right)^{2} .
$$

The total variation across municipalities was quantified by standard deviation $s_{\mathrm{T}}$ :

$$
s_{\mathrm{T}}^{2}=\frac{1}{M-1} \sum_{m=1}^{M}\left(\theta_{m}-\bar{\theta}\right)^{2},
$$

where $\theta_{m}=\beta_{r_{m}}+v_{m}+u_{m}$ and $\bar{\theta}$ is the arithmetic mean of $\theta_{m}$.

Variation in the age-standardised 5-year net survival was quantified by standard deviations $s_{\mathrm{SB}}, s_{\mathrm{SW}}$ and $s_{\mathrm{ST}}$ :

$$
\begin{aligned}
& s_{\mathrm{SB}}^{2}=\frac{1}{R-1} \sum_{r=1}^{R} D_{\mathrm{S}}(5, r, \cdot)^{2}, \quad s_{\mathrm{SW}}^{2}=\frac{1}{M-1} \sum_{m=1}^{M} D_{\mathrm{S}}(5, \cdot, m)^{2} \text { and } \\
& s_{\mathrm{ST}}^{2}=\frac{1}{M-1} \sum_{m=1}^{M} D_{\mathrm{S}}\left(5, r_{m}, m\right)^{2}
\end{aligned}
$$

where $D_{\mathrm{S}}(5, r, \cdot)=S_{\mathrm{E}}(5, r, \cdot)-\frac{1}{R} \sum_{r} S_{\mathrm{E}}(5, r, \cdot)$ is the hospital district specific deviation of the 5-year net survival from the arithmetic mean over $R$ hospital districts, $D_{\mathrm{S}}(5, \cdot, m)=S_{\mathrm{E}}(5, \cdot, m)-\frac{1}{M} \sum_{m} S_{\mathrm{E}}(5, \cdot, m)$ and $D_{\mathrm{S}}(5, r, m)=$ $S_{\mathrm{E}}(5, r, m)-\frac{1}{M} \sum_{m} S_{\mathrm{E}}(5, r, m)$ are the municipality-specific deviations from the arithmetic means over $M$ municipalities, and $S_{\mathrm{E}}(5, r, \cdot)$ and $S_{\mathrm{E}}(5, \cdot, m)$ are calculated by setting $u_{m}=v_{m}=0$ and $\beta_{r}=0$, respectively.

\subsection{Approximation and simulation of posterior distribution}

The model was implemented in a Bayesian framework using both the INLA approach and the MCMC simulation. Vague $\mathrm{N}(0,1000)$ priors were assumed for the fixed effects $\boldsymbol{\alpha}$. For the standard deviation parameters $\sigma_{\beta}, \sigma_{u}$ and $\sigma_{v}$ of the regional random effects, we assumed half-normal distributions centered at 0 with standard deviation 100. A half-normal and a uniform distribution were recommended by Gelman and Hill [36] for a diffuse but a proper prior distribution of hierarchical standard deviations and are expected to work well in general, unless the number of regions is below 5 . The half-normal 
prior was convenient for the comparison between the two approaches by using R-INLA and OpenBUGS, because it is available in both programs. For a formal discussion of weakly informative priors in this context, see articles by Simpson et al[37] and Riebler et al.[38]

The joint posterior distribution is given by

$p\left(\boldsymbol{\alpha}, \boldsymbol{\beta}, \boldsymbol{u}, \boldsymbol{v}, \sigma_{\beta}, \sigma_{u}, \sigma_{v} \mid \boldsymbol{Y}\right) \propto L(\boldsymbol{\alpha}, \boldsymbol{\beta}, \boldsymbol{u}, \boldsymbol{v}) p(\boldsymbol{\alpha}) p\left(\boldsymbol{\beta} \mid \sigma_{\beta}\right) p\left(\boldsymbol{u} \mid \sigma_{u}\right) p\left(\boldsymbol{v} \mid \sigma_{v}\right) p\left(\sigma_{\beta}\right) p\left(\sigma_{u}\right) p\left(\sigma_{v}\right)$,

where $L$ is the Poisson likelihood of the entire patient population based on collapsed data $\boldsymbol{Y}=\left(\boldsymbol{d}, \boldsymbol{d}^{*}, \boldsymbol{y}\right)$ stratified by municipality $m$, age group $g$ and follow-up time interval $j$ :

$\ln [L(\boldsymbol{\alpha}, \boldsymbol{\beta}, \boldsymbol{u}, \boldsymbol{v})]=\sum_{m g j}\left\{d_{m g j} \ln \left[d_{m g j}^{*} / y_{m g j}+\nu(\boldsymbol{\alpha}, \boldsymbol{\beta}, \boldsymbol{u}, \boldsymbol{v})\right]-\nu(\boldsymbol{\alpha}, \boldsymbol{\beta}, \boldsymbol{u}, \boldsymbol{v}) y_{m g j}\right\}+$ constant

\subsubsection{MCMC simulation}

MCMC is a simulation-based approach for computations in Bayesian inference by generating a sample of correlated values from the posterior distribution by running a Markov chain. Gibbs sampling is an MCMC algorithm that generates samples of each parameter conditional on all the other parameters. It is used, for instance, by OpenBUGS [39] and JAGS [40] and can be easily applied to the inference of complex models that include a large number of parameters. The well known problem with Gibbs sampling is that subsequent values in MCMC simulation are highly correlated in hierarchical models, because the random effects strongly depend on each other and also on the standard deviations of their distributions. Alternative approaches to overcome these problems include construction of a joint proposal for the full conditional distribution of random effects and updating random effect and hierarchical parameters jointly. Yet, despite these developments MCMC sampling remains painfully slow from the end user's point of view.[25]

In our application, the MCMC simulations were carried out by Gibbs sampling using OpenBUGS (version 3.2.3).[39, 41] Three series of 4200000 iterations were run. The first 200000 iterations were discarded as burn-in in each run. Every 100th and 2000th of the remaining iterations was stored in the analyses without and with municipality-specific effects, respectively. The posterior inference was based on the pooled sample of 120000 (analyses without municipalities) and 6000 (municipality-specific analyses) thinned values.

The coda package in $\mathrm{R}$ was used for the convergence diagnostic of the Markov chain and for the estimation of the Monte Carlo error. The convergence was examined by calculating the Gelman-Rubin statistic, as modified by Brooks and Gelman.[42] The OpenBUGS program code for specifying the model in the municipality-specific analyses is included in the supplementary material. 


\subsubsection{INLA approximation}

As an alternative to simulation-based MCMC, INLA is a deterministic approach proposed by Rue et al[25] for computations in Bayesian inference on the class of latent Gaussian models which include a wide variety of commonly applied statistical models.[27] It produces accurate and fast approximations to the marginal posterior distributions based on Laplace approximations and numerical integration. The INLA approach is implemented in R-INLA package in R.[25]

In the INLA approach, the posterior marginal distributions $p\left(\theta_{q} \mid \boldsymbol{Y}\right)$ and $p\left(\psi_{q} \mid \boldsymbol{Y}\right)$ of parameters $\boldsymbol{\theta}=(\boldsymbol{\alpha}, \boldsymbol{\beta}, \boldsymbol{u}, \boldsymbol{v})$ and hyperparameters $\boldsymbol{\psi}=\left(\sigma_{\beta}, \sigma_{u}, \sigma_{v}\right)$, respectively, are approximated by

$$
\begin{aligned}
\tilde{p}\left(\theta_{q} \mid \boldsymbol{Y}\right) & =\int \tilde{p}\left(\theta_{q} \mid \boldsymbol{\psi}, \boldsymbol{Y}\right) \tilde{p}(\boldsymbol{\psi} \mid \boldsymbol{Y}) \mathrm{d} \boldsymbol{\psi} \quad \text { and } \\
\tilde{p}\left(\psi_{q} \mid \boldsymbol{Y}\right) & =\int \tilde{p}(\boldsymbol{\psi} \mid \boldsymbol{Y}) \mathrm{d} \boldsymbol{\psi}_{-q}
\end{aligned}
$$

where subscript $q$ refers to the $q$ th component of the correspoding vector $(\boldsymbol{\theta}$ or $\boldsymbol{\psi})$ and $-q$ to the vector without the $q$ th component. The Laplace approximation of the joint posterior of the hyperparameters is denoted by $\tilde{p}(\boldsymbol{\psi} \mid \boldsymbol{Y})$ and the simplified Laplace approximation was used for $\tilde{p}\left(\theta_{q} \mid \boldsymbol{\psi}, \boldsymbol{Y}\right)$. The first integral (2) can be solved numerically through a finite weighted sum

$$
\tilde{p}\left(\theta_{q} \mid \boldsymbol{Y}\right) \approx \sum_{k} \tilde{p}\left(\theta_{q} \mid \boldsymbol{\psi}^{(k)}, \boldsymbol{Y}\right) \tilde{p}\left(\boldsymbol{\psi}^{(k)} \mid \boldsymbol{Y}\right) \Delta_{k}
$$

over some relevant integration points $\left\{\boldsymbol{\psi}^{(k)}\right\}$ with a corresponding set of weights $\left\{\Delta_{k}\right\}$. [25, 43] Rue et al[25] proposed two different strategies to identify the integration points for exploring the joint posterior of the hyperparameters $\tilde{p}(\boldsymbol{\psi} \mid \boldsymbol{Y})$. In the grid integration strategy the joint posterior is evaluated in a regular grid of points. The other one is called central composite design (CCD) strategy and reduces the computational costs by using much fewer integration points, these being located on a sphere around the mode.[27]

In addition to providing approximations to posterior marginals of model parameters $\theta_{q}$ and $\psi_{q}$, R-INLA includes tools to draw samples independently from an approximated full posterior distribution that can be used to estimating the functionals of model parameters $\boldsymbol{\theta}$. The sampling makes use of the values $\tilde{p}\left(\boldsymbol{\psi}^{(k)} \mid \boldsymbol{Y}\right)$ of the joint posterior of hyperparameters and the joint Gaussian approximation of $\tilde{p}\left(\boldsymbol{\theta} \mid \boldsymbol{\psi}^{(k)}, \boldsymbol{Y}\right)$ that were already computed for the grid points $\left\{\boldsymbol{\psi}^{(k)}\right\}$ in the numerical integration (3). The sampling algorithm has two steps:

1. Sample hyperparameters $\boldsymbol{\psi}^{*}$ from the set of $\left\{\boldsymbol{\psi}^{(k)}\right\}$ with probabilities $\tilde{p}\left(\boldsymbol{\psi}^{(k)} \mid \boldsymbol{Y}\right) \Delta_{k}$ computed in the numerical integration (3).

2. Conditional on the sampled $\boldsymbol{\psi}^{*}$, sample $\boldsymbol{\theta}^{*}$ jointly from the corresponding joint Gaussian approximation of $p\left(\boldsymbol{\theta} \mid \boldsymbol{\psi}^{*}, \boldsymbol{Y}\right)$ which was already 
calculated in the numerical integration (3) in order to obtain the approximation $\tilde{p}\left(\boldsymbol{\psi}^{*} \mid \boldsymbol{Y}\right)$ to the joint posterior of the hyperparameters. Here, however, an improved approximation using a Gaussian copula [44] that retains the dependence structure of the Gaussian approximation of $p\left(\boldsymbol{\theta} \mid \boldsymbol{\psi}^{*}, \boldsymbol{Y}\right)$ while having the improved approximations of the marginals $p\left(\theta_{q} \mid \boldsymbol{\psi}^{*}, \boldsymbol{Y}\right)$ is used.

In our application, the model was fitted by using inla function in R-INLA package [25] with the default CCD integration strategy. Then, the grid integration strategy was utilised to improve the estimates of the posterior marginals of the hyperparameters by using inla.hyperpar function. The posterior estimates were based on a sample of 3000 values drawn independently from the approximated posterior distribution by using the function inla.posterior.sample. The program code for fitting the model in the municipality-specific analyses is included in the supplementary material.

\section{Results}

\subsection{Computation time}

In the MCMC approach, computation time was based on the number of iterations required to obtain the Monte Carlo (MC) errors of $5 \%$ or less out of the standard deviations of target parameters $s_{\mathrm{B}}, s_{\mathrm{W}}$ and $s_{\mathrm{T}}$ : 260 thousands and 1.1 million iterations in the analyses without and with municipality-specific effects, respectively. In R-INLA, parallel computing with four threads was utilised.

In the model without municipality-specific effects, the analyses of ovary and breast cancer took altogether 2.1 minutes with the INLA and 100 minutes with the MCMC approach (based on the 5\% level of MC error). When the effects of municipalities were included in the model, computation time increased to 1.6 hours and 400 hours in the INLA and the MCMC approach, respectively. If the number of threads was limited to one in R-INLA, the analyses took 3.25 hours. In the INLA approach, the posterior sampling part took $61 \%$ and $29 \%$ out of the total computation time in the models without and with municipality-specific effects, respectively.

In the following, results are based on the total number of stored iterations with MC errors of $1.4 \%$ or less out of the standard deviations of the parameters.

\subsection{Regional variation}

Figure 1 compares the estimates of the standard deviations $s_{\mathrm{B}}$ and $s_{\mathrm{SB}}$ between the INLA and the MCMC approach in the model without municipalityspecific effects. The posterior means and $95 \%$ posterior intervals were very 
similar between the two approaches. In ovarian cancer, the standard deviations were relatively large in 1965-1969 and in 2000-2004. In breast cancer, the standard deviations decreased from the 1960s to the 1980s. From 1990s, the variation in the relative excess mortality has not decreased even though it has decreased on the scale of 5-year net survival.

Figure 2 compares the estimates of the standard deviations $s_{\mathrm{B}}, s_{\mathrm{W}}$ and $s_{\mathrm{T}}$ between the INLA and the MCMC approach in the model in which the effects of municipalities are also included. The estimates of INLA are close to those of MCMC simulation. The standard deviation $s_{\mathrm{B}}$ is smaller than that based on model without municipality. Variation within hospital districts dominated in the total variation. We estimated the average of variation over the last three 5-year periods for obtaining a more precise estimate. In 20002014 , the average of variation $s_{\mathrm{W}}^{2}$ within hospital districts was $68 \%(95 \%$ posterior interval: $35-93 \%)$ and $82 \%(60-98 \%)$ out of the total variation $s_{\mathrm{T}}^{2}$ in ovarian and breast cancer, respectively. Supplementary Figure S2 shows the standard deviations in the 5-year net survival.

The close similarity in the estimates of standard deviations between INLA and MCMC (Figures 1 and 2) was based on the improved estimates obtained by using the grid integration strategy in INLA. By using the CCD integration in INLA, the posterior means were overestimated on average by $15 \%$ and the posterior intervals were up to $29 \%$ shorter, because the lower limits of the intervals were clearly overestimated.

Although the estimates of the standard deviations were similar, the posterior means of the baseline of 5-year net survival based on INLA was most often smaller than that based on MCMC. The largest difference was 2.2 percentage points (pp) in the municipality-specific analysis of ovarian cancer in 1960-64 (Table 1). In the analysis without municipality (Table S1), the differences between INLA and MCMC were on average 33\% smaller than those in municipality-specific analysis. The regional estimates of 5-year net survival based on the INLA approach are shown in Figure 3. In ovarian cancer, there was more variation between hospital districts in the end of 1960s and in the beginning of 2000s. However, the capital of Finland was divergent in 2000s but not in 1960s. Figure 4 shows the regional estimates of 5 -year net survival in 1960-1964 and in 2010-2014 divided by hospital districts in order to assess variation within each hospital district.

\subsection{Simulation study on bias in estimators of baseline excess mortality}

A simulation study was performed to compare the INLA and the MCMC approach in the estimation of baseline excess mortality $\nu_{i j}=\exp \left(\boldsymbol{\alpha}^{\prime} \boldsymbol{x}_{i j}\right)$, because the baseline estimates differed between the approaches. Altogether 1000 datasets were generated from the Poisson distribution by using the observed numbers of deaths in ovarian and breast cancer patients as the expected values in each stratum of age group, follow-up time interval and calendar time period (categorised similarly as earlier in Sections 2.1 and 2.2). 
Excess mortality was summarised in the terms of the net survival index. The MCMC and the INLA estimators of 5-year net survival were compared with the maximum likelihood estimator (MLE) in two different baseline models:

M1) a full model which included a parameter for each stratum of age group and follow-up time, and

M2) a simplified model in Section 2.2, which assumed the same interaction effect of age and follow-up time in the intervals of follow-up time from 1 to 5 years.

The bias, variance and mean squared error were calculated and, in the INLA and the MCMC approach, were based on the posterior means. The MCMC approach was carried out using JAGS program (version 4.1.0) [40] and was based on 110000 iterations of which the first 10000 samples were discarded as burn-in.

The expectation of MLE was between the expectations of INLA and MCMC in the simulation setting of ovarian and breast cancer (Supplementary Figures S3-S6). In the age-standardised 5-year net survival, the absolute differences between INLA and MLE were about the same as those between MCMC and MLE.

In the full baseline model (M1), from which the datasets were generated, the MLE was practically unbiased (Supplementary Figures S3 and S5). In ovarian cancer, the MCMC approach overestimated the age-standardised net survival on average by 0.8 percentage points (pp), whereas the INLA approach underestimated the survival on average by $1.6 \mathrm{pp}$. In breast cancer, MCMC overestimated and INLA underestimated the survival on average by $0.4 \mathrm{pp}$ and $0.6 \mathrm{pp}$, respectively.

In the simplified baseline model (M2), also the MLE was slightly biased (Supplementary Figures S4 and S6), because the datasets were generated on the basis of observed mortality rates. The magnitudes of bias related to the MCMC and the INLA approach were clearly smaller than those in the full baseline model, especially in ovarian cancer. MCMC overestimated the net survival on average by $0.2 \mathrm{pp}$ on ovarian and $0.1 \mathrm{pp}$ in breast cancer. INLA underestimated the corresponding quantities on average by $0.3 \mathrm{pp}$ and 0.2 pp.

In the age group specific estimators of the 5-year net survival, the INLA approach introduced some bias in every age group whereas the MCMC approach was biased mainly in the two oldest age groups. The largest values of bias were related to small number of deaths $\left(d_{m g j}\right)$ or excess deaths $\left(d_{m g j}-d_{m g j}^{*}\right)$ in an interval of follow-up time. The posterior distribution of the logarithm of excess mortality based on the MCMC simulations had a heavy lower tail, if the number of excess deaths was small. This resulted in the overestimation of net survival. On the other hand, the posterior approximation based on the INLA was symmetric and poor (supporting large values of excess mortality), if the number of deaths was small, and especially, if it was smaller than the expected number $d_{m g j}^{*}$. This resulted in the underestimation of net survival. 


\section{Discussion}

In this study we demonstrated and validated the use of the INLA approach for estimation of regional variation in excess mortality and relative or net survival. The study showed that the estimates of regional variation were similar based on the INLA and the MCMC simulations, and the results were obtained much faster by using the INLA than the MCMC approach. The simulation study based on the model without random effects showed that both approaches introduced some bias in the estimation of baseline excess mortality, but the magnitude of bias was most often modest.

Small variation in survival between hospital districts does not imply regional equity, because there may be substantial variation within hospital districts. The variation within hospital ditricts is influenced by differences in personal health behaviour and in primary health care. The former one includes person's ability to seek medical care and person's health status which affects the available treatment options. The latter one includes the availability of health care services and delays in the patient path from primary care to the central hospital.

The same determinants that cause variation in survival within hospital ditricts cause variation between hospital districts, if the distributions of the determinants differ between hospital districts. In addition to these determinants, variation between hospital districts is affected by differences in cancer care provided by the central hospitals and university hospitals.

Spatial correlation in survival across municipalities was considered important, as it is likely that many determinants of survival exist that correlate between neighbouring municipalities. Information on geographical proximity between hospital districts was not incorporated in the models, because patients in a given municipality are treated in a designated central hospital and further referred to the pertinent university hospital, if more advanced care is needed.

Patients' mortality was compared to the population mortality in the corresponding hospital district. Although the reference population also includes cancer patients under study, this can usually be considered to have a negligible effect on the estimated net survival.[45] In prostate cancer and especially in all cancer sites combined, an adjustment for the reference population mortality has been recommended.[46, 47] This adjustment was not done, for example, in the EUROCARE-5 and the CONCORD-3 study,[5, 6] either. In our application, the 5-year net survival is likely to be overestimated approximately by 0.7 percentage points in breast cancer and less than that in ovarian cancer according to the results of Talbäck and Dickman.[46]

Considering hospital district-specific population mortality rates to be fixed without random variation was shown to be a reasonable assumption for the estimation of excess mortality.[7] However, variation in excess mortality within hospital districts includes variation due to potential differences in population mortality between municipalities within each hospital district. In 
municipality-specific population mortality rates, random variation should be taken into account by using an extended model that includes the likelihood for population mortality.[7]

Standard deviations $s_{\mathrm{B}}, s_{\mathrm{W}}$ and $s_{\mathrm{T}}$ quantify how much there is variability in the logarithm of relative excess mortality across regions. This variation can remain at the same level, even though regional variation in net survival decreases, as it happened in breast cancer in Finland. Interpretation of both measures depends on the average level of excess mortality and net survival. The choice of the measure is ultimately based on the decision on whether we shall count the failures or the successes and whether we shall make relative or absolute comparisons. [48] The standard deviations $s_{\mathrm{SB}}, s_{\mathrm{SW}}$ and $s_{\mathrm{ST}}$ of net survival may have more attractive interpretation, because net survival is commonly used for summarising excess mortality among cancer patients. [5, 6]

An increased regional variation in the survival of ovarian cancer around year 2000 was shown by Pohar Perme et al[49] in the study that assessed variation between the five cancer control regions in Finland in 10-year periods of diagnosis from 1953-1962 to 1993-2002. Our study showed that the increased difference was more related to the variation between the hospital districts than to the variation within them, and that the regional variation decreased thereafter.

The decrease in the standard deviation of the net survival in breast cancer does not indicate that the public health importance of regional variation has decreased, because breast cancer incidence increased simultaneously. When assessing the public health importance, the changes in incidence can be taken into account, for example by estimating potential numbers of person years or deaths that would be saved, if regional variation were eliminated.[7]

We estimated the finite population variances, because the data were collected from all the 21 hospital districts and 313 municipalities in Finland and we wish to describe the variation across these very districts. Alternatively, variation may be measured by estimating the so-called superpopulation variance, i.e. the theoretical variance of the distribution of the random effects, would be appropriate for assessing the uncertainty about the value of a potential new district not in the original set.[36] The posterior mean of the superpopulation standard deviation $\sigma_{\beta}$ was on average $7 \%$ larger than that of the finite population $s_{\mathrm{B}}$. The credible intervals for $\sigma_{\beta}$ were from $14 \%$ to $27 \%$ wider than those of $s_{\mathrm{B}}$. The superpopulation variance of municipalities is not directly obtained, because the standard deviation $\sigma_{u}$ of the CAR model is not comparable with that $\sigma_{v}$ of the unstructured effect. However, the intrinsic CAR model can be scaled, such that the conditional variance will be equal to the marginal one.[50]

Censoring all follow-up times at 5 years may affect the results of regional variation if notable excess mortality exists and varies across regions after the first 5 years since diagnosis. Quite often follow-up is considered only up to the first 5 years, because thereafter, excess mortality typically becomes small. Among breast cancer patients, however, the mortality remains elevated even 
beyond 5 years compared with the reference population,[51] and therefore, including a longer-term follow-up could have an effect on regional variation.

Excess hazard was assumed to be constant in each follow-up time interval according to model ??. We divided the first year of follow-up into three and nine-months intervals, because excess mortality changed most rapidly in the beginning of follow-up especially among ovarian cancer patients. In addition to incorrectly specified functional form, the model may suffer from lack-of-fit due to omission of important unmeasured covariates (i.e. overdispersion) or absence of important interaction terms.[15] Overdispersion may occur, if important determinants of cancer survival are omitted in the model. For example, tumour stage and patient's health status at the time of diagnosis and information on whether a patient participated in mammography screening are such covariates that may cause overdispersion. According to a score test for overdispersion proposed by Luque-Fernandez et al,[52] no statistically discernible overdispersion could be observed for all periods combined. However, some evidence for overdispersion was found in some periods: 1990-1994 in breast and ovarian cancer and in 2005-2009 in ovarian cancer (analyses without municipality); and 1990-1994 and 2000-2004 in breast cancer (municipality-specific analyses). In addition, we did a sensitivity check by including an additional stratum-specific random effect in the model to adjust for potential overdispersion. The effect of adjusting for overdispersion was small. By including the overdispersion effect in the model without municipalities, posterior mean of $s_{B}$ decreased on average by $3 \%$ in ovarian cancer and by $5 \%$ in breast cancer and the length of the posterior interval increased by $3 \%$ and by $5 \%$, respectively. In the municipality-specific model, the overdispersion-adjusted estimates differed from the non-adjusted ones less than $3 \%$.

Stratifying the calendar periods into 5-year periods and modelling them separately was a convenient choice, because the intrinsic CAR model was available in R-INLA and OpenBUGS. It would be difficult to select particular break points at which some actions related to cancer survival occurred. In addition, a temporal stucture that would remain valid over the long 55 years calendar period, was not plausible. Spatio-temporal models become easily complex, and the challenge is to find a sufficiently realistic, yet manageable model that includes necessary interactions. R-INLA includes spatiotemporal models with a first- or second-order random walk structure for the temporal effect and several options for space-time interactions. [43, 53] We also tried to fit a spatio-temporal model where we assumed a second-order random walk across the 11 calendar periods for each hospital district independently. In addition, we tried to fit another spatio-temporal model where the temporal dependence stucture of each municipality depends on the temporal patterns of the neighbouring municipalities. However, we did not succeed to fit the models with municipality-specific effects due to memory limitations in our computation environment. The only model that converged (without the grid integration strategy in INLA), was a model without municipalityspecific effects in breast cancer. There is ongoing work within the R-INLA project to improve this limitation.

INLA has shown to give very accurate approximations to the posterior 
marginals of the model parameters over a wide range of commonly used latent Gaussian models including generalised linear mixed models.[25, 54] In this study, we were interested in the posterior estimates of functionals of model parameters rather than the posterior marginal distributions of the parameters. For example, presenting variation on the scale of net survival requires the joint posterior of the all model parameters except the variance parameters. In our application, the sampling algorithm implemented in RINLA package for generating samples from an approximated posterior proved to be very accurate to estimating these functionals.

INLA and MCMC approaches introduced some bias in the estimation of the baseline excess mortality. The model itself may introduce bias, because it does not allow negative excess mortality. However, because the maximum likelihood estimator was practically unbiased, the bias in the MCMC approach relates to the prior assumption that becomes informative because of the model and its likelihood, which is greater than 0 for excess mortality $\nu=0$. Therefore, a flat normal prior for the logarithm of excess mortality may result in posterior $p[\ln (\nu) \mid \boldsymbol{Y}]$ with a heavy negative skew, if the number of deaths is small, because small values of excess mortality are supported by the prior. The INLA approximation, on the other hand, may not catch the shape and the spread of the negatively skewed posterior and may therefore support too large values of excess mortality. Results based on the simplified baseline model (M2) show that the bias may be reduced by excluding some interaction terms between follow-up time and age, if the simplified model still fits the data well. The previous study of Saez et al[22] that used a similar model and MCMC simulation did not explore the potential bias. The model included the main effects of follow-up time and age only. This is, however, often invalid assumption in cancer survival, and therefore, may not reduce bias.

In the MCMC estimation, the convergence has to be explored carefully. In our application, the mixing of the Markov chain was very slow especially in the municipality-specific effects and their standard deviations. A large number of iterations were run in order to achieve negligible Monte Carlo errors. A clearly smaller number of iterations satisfied the $5 \%$ level of MC error suggested in the OpenBUGS manual,[39] but computation time was still from 48-fold to 250-fold in the MCMC approach compared to the INLA approach. This is in line with other applications of hierarchical Poisson model implemented in R-INLA and OpenBUGS [55] or WinBUGS,[53] as these other implemantations did not utilise the grid integration and the posterior sampling of INLA that were necessary in our application.

In the estimation of regional variation in excess mortality of ovarian and breast cancer patients in Finland, the INLA approach was accurate, fast and easy to implement by using the R-INLA package. It can be recommended for routine use in monitoring regional variation in excess mortality and net survival. 


\section{Acknowledgements}

Karri Seppä was supported by a grant from the Finnish Cancer Foundation (to J. Pitkäniemi).

\section{References}

[1] S. Karjalainen, "Geographical variation in cancer patient survival in finland: chance, confounding, or effect of treatment?," Journal of Epidemiology and Community Health, vol. 44, pp. 210-214, 1990.

[2] J. Estève, E. Benhamou, M. M. Croasdale, and L. Raymond, "Relative survival and the estimation of net survival: elements for further discussion," Statistics in Medicine, vol. 9, pp. 529-538, 1990.

[3] M. Pohar Perme, J. Stare, and J. Estève, "On estimation in relative survival," Biometrics, vol. 68, pp. 113-120, 2012.

[4] K. Seppä, T. Hakulinen, E. Läärä, and J. Pitkäniemi, "Comparing net survival estimators of cancer patients," Statistics in Medicine, vol. 35, pp. 1866-1879, 2016.

[5] R. De Angelis, M. Sant, M. P. Coleman, S. Francisci, P. Baili, D. Pierannunzio, A. Trama, O. Visser, H. Brenner, E. Ardanaz, M. Bielska-Lasota, G. Engholm, A. Nennecke, S. Siesling, F. Berrino, and R. E.-. W. G. Capocaccia, "Cancer survival in europe 1999-2007 by country and age: results of eurocare-5 - a population-based study," Lancet Oncology, vol. 15, pp. 23-34, 2014.

[6] C. Allemani, T. Matsuda, V. Di Carlo, R. Harewood, M. Matz, M. Nik` sić, A. Bonaventure, M. Valkov, C. Johnson, J. Estève, O. Ogunbiyi, G. Azevedo E Silva, W. Chen, S. Eser, G. Engholm, C. Stiller, A. Monnereau, R. Woods, O. Visser, G. Lim, H. Weir, and M. C. W. G. Coleman, "Global surveillance of trends in cancer survival 2000-14 (concord-3): analysis of individual records for 37513025 patients diagnosed with one of 18 cancers from 322 populationbased registries in 71 countries," The Lancet, vol. 391, pp. 1023-1075, 2018.

[7] K. Seppä, T. Hakulinen, and E. Läärä, "Regional variation in relative survival - quantifying the effects of the competing risks of death by using a cure fraction model with random effects," Journal of the Royal Statistical Society, Series C, vol. 63, pp. 179-190, 2014.

[8] K. D. Skyrud, F. Bray, M. T. Eriksen, Y. Nilssen, and B. Møller, "Regional variations in cancer survival: Impact of tumour stage, socioeconomic status, comorbidity and type of treatment in norway," International Journal of Cancer, vol. 138, pp. 2190-2200, 2016.

[9] A. Pokhrel, P. Martikainen, E. Pukkala, M. Rautalahti, K. Seppä, and T. Hakulinen, "Education, survival and avoidable deaths in cancer patients in finland," British Journal of Cancer, vol. 103, pp. 1109-1114, 2010.

[10] C. P. Nelson, P. C. Lambert, I. B. Squire, and D. R. Jones, "Flexible parametric models for relative survival, with application in coronary heart disease," Statistics in Medicine, vol. 26, pp. 5486-5498, 2007.

[11] O. A. Alabas, C. P. Gale, M. Hall, M. J. Rutherford, K. Szummer, S. Lawesson, J. Alfredsson, B. Lindahl, and T. Jernberg, "Sex differences in treatments, relative survival, and excess mortality following acute myocardial infarction: National cohort study using the swedeheart registry," Journal of the American Heart Association, vol. 6, p. e007123, 2017. 
[12] T. Huttunen, M. von und zu Fraunberg, T. Koivisto, A. Ronkainen, J. Rinne, R. Sankila, K. Seppä, and J. Jääskeläinen, "Long-term excess mortality of 244 familial and 1502 sporadic one-year survivors of aneurysmal subarachnoid hemorrhage compared with a matched eastern finnish catchment population," Neurosurgery, vol. 68, pp. 20-27, 2011.

[13] K. Bhaskaran, O. Hamouda, M. Sannes, F. Boufassa, A. Johnson, P. Lambert, and K. C. C. Porter, "Changes in the risk of death after hiv seroconversion compared with mortality in the general population," JAMA, vol. 300, pp. 5159, 2008.

[14] T. Hakulinen and L. Tenkanen, "Regression analysis of relative survival rates," Applied Statistics, vol. 36, pp. 309-317, 1987.

[15] P. W. Dickman, A. Sloggett, M. Hills, and T. Hakulinen, "Regression models for relative survival," Statistics in Medicine, vol. 23, pp. 51-64, 2004.

[16] R. De Angelis, R. Capocaccia, T. Hakulinen, B. Soderman, and A. Verdecchia, "Mixture models for cancer survival analysis: application to population-based data with covariates," Statistics in Medicine, vol. 18, pp. 441-454, 1999.

[17] P. D. Sasieni, "Proportional excess hazards," Biometrika, vol. 83, pp. 127-141, 1987.

[18] M. Pohar Perme, R. Henderson, and J. Stare, "An approach to estimation in relative survival regression," Biostatistics, vol. 10, pp. 136-146, 2009.

[19] O. Kuss, T. Blankenburg, and J. Haerting, "A relative survival model for clustered responses," Biometrical Journal, vol. 50, pp. 408-418, 2008.

[20] L. Fairley, D. Forman, R. West, and S. Manda, "Spatial variation in prostate cancer survival in the northern and yorkshire region of england using bayesian relative survival smoothing," British Journal of Cancer, vol. 99, pp. 1786-1793, 2008.

[21] S. M. Cramb, K. L. Mengersen, and P. D. Baade, Atlas of Cancer in Queensland: geographical variation in incidence and survival 1998 to 200\%. Brisbane, Queensland: Viertel Centre for Research in Cancer Control, Cancer Council Queensland; 2011.

[22] M. Saez, M. A. Barceló, C. Martos, C. Saurina, R. Marcos-Gragera, G. Renart, R. Ocaña Riola, C. Feja, and T. Alcalá, "Spatial variability in relative survival from female breast cancer," Journal of the Royal Statistical Society, Series A, vol. 175, pp. 107-134, 2012.

[23] S. Y. Kang, S. M. Cramb, N. M. White, S. J. Ball, and K. L. Mengersen, "Making the most of spatial information in health: a tutorial in bayesian disease mapping for areal data," Geospatial Health, vol. 11, p. 428, 2016.

[24] S. M. Cramb, K. L. Mengersen, P. C. Lambert, L. M. Ryan, and P. D. Baade, "A flexible parametric approach to examining spatial variation in relative survival," Statistics in Medicine, vol. 35, pp. 5448-5463, 2016.

[25] H. Rue, S. Martino, and N. Chopin, "Approximate bayesian inference for latent gaussian models using integrated nested laplace approximations (with discussion)," Journal of the Royal Statistical Society, Series B., vol. 71, pp. 319-392, 2009.

[26] T. G. Martins, D. Simpson, F. Lindgren, and H. Rue, "Bayesian computing with inla: New features," Computational Statistics 83 Data Analysis, vol. 67, pp. 68-83, 2013.

[27] H. Rue, A. Riebler, S. H. Sørbye, J. B. Illian, D. P. Simpson, and F. K. Lindgren, "Bayesian computing with inla: A review," Annual Review of Statistics and Its Applications, vol. 4, pp. 395-421, 2017. 
[28] Ministry of Social Affairs and Health, "Health care in finland," Brochures of the Ministry of Social Affairs and Health 2eng, 2013. URL: www.urn.fi/URN:ISBN:978-952-00-3395-8 (accessed March 27, 2018).

[29] H. Brenner and O. Gefeller, "An alternative approach to monitoring cancer patient survival," Cancer, vol. 78, pp. 2004-2010, 1996.

[30] "Surveillance research program, national cancer institute seer*stat software. url: seer.cancer.gov/seerstat (accessed march 27, 2018),"

[31] I. Corazziari, M. Quinn, and R. Capocaccia, "Standard cancer patient population for age standardising survival ratios," European Journal of Cancer, vol. 40, pp. 2307-2316, 2004.

[32] J. Besag, J. York, and A. Mollie, "Bayesian image restoration, with two applications in spatial statistics," Annals of the Institute of Statistical Mathematics, vol. 43, pp. 1-59, 1991 .

[33] D. Lee, "A comparison of conditional autoregressive models used in bayesian disease mapping," Spatial and Spatio-temporal Epidemiology, vol. 2, pp. 79-89, 2011.

[34] A. Freni-Sterrantino, M. Ventrucci, and H. Rue, "A note on intrinsic conditional autoregressive models for disconnected graphs," Spatial and Spatiotemporal Epidemiology, vol. 26, pp. 25-34, 2018.

[35] P. C. Lambert, P. W. Dickman, and M. J. Rutherford, "Comparison of different approaches to estimating age standardized net survival," BMJ Medical Research Methodology, vol. 15, p. 64, 2015.

[36] A. Gelman and J. Hill, Data Analysis Using Regression and Multilevel/Hierarchical Models. New York: Cambridge University Press; 2007.

[37] D. P. Simpson, H. Rue, A. Riebler, T. G. Martins, and S. H. Sørbye, "Penalising model component complexity: A principled, practical approach to constructing priors (with discussion)," Statistical Science, vol. 32, pp. 1-28, 2017.

[38] A. Riebler, S. H. Sørbye, D. Simpson, and H. Rue, "An intuitive Bayesian spatial model for disease mapping that accounts for scaling," Statistical Methods in Medical Research, vol. 25, pp. 1145-1165, 2016.

[39] D. Spiegelhalter, A. Thomas, N. Best, and D. Lunn OpenBUGS User Manual, Version 3.2.3., March 2014. URL: www.openbugs.net (accessed January 11, 2018).

[40] M. Plummer JAGS Version 3.4.0 user manual, 2013. URL: mcmcjags.sourceforge.net (accessed March 27, 2018).

[41] D. Lunn, D. Spiegelhalter, A. Thomas, and N. Best, "The bugs project: Evolution, critique, and future directions," Statistics in Medicine, vol. 28, pp. 30493067, 2009.

[42] S. P. Brooks and A. Gelman, "General methods for monitoring convergence of iterative simulations," Journal of Computational and Graphical Statistics, vol. 7, pp. 434-455, 1998.

[43] M. Blangiardo and M. Cameletti, Spatial and Spatio-temporal Bayesian Models with R-INLA. John Wiley \& Sons; 2015.

[44] E. Ferkingstad and H. Rue, "Improving the inla approach for approximate bayesian inference for latent gaussian models," Electronic Journal of Statistics, vol. 9, pp. 2706-2731, 2015.

[45] F. Ederer, L. M. Axtell, and S. J. Cutler, "The relative survival rate: a statistical methodology," National Cancer Institute Monograph, vol. 6, pp. 101-121, 1961. 
[46] M. Talbäck and P. W. Dickman, "Estimating expected survival probabilities for relative survival analysis - exploring the impact of including cancer patient mortality in the calculations," European Journal of Cancer, vol. 47, pp. 2626$2632,2011$.

[47] S. R. Hinchliffe, P. W. Dickman, and P. C. Lambert, "Adjusting for the proportion of cancer deaths in the general population when using relative survival: a sensitivity analysis," Cancer Epidemiology, vol. 36, pp. 148-152, 2012.

[48] M. C. Sheps, "Shall we count the living of the dead?," New England Journal of Medicine, vol. 259, pp. 1210-1214, 1958.

[49] M. Pohar Perme, T. Hakulinen, M. Jesenko, R. Sankila, and J. Stare, "Has equity in relative survival improved over time in finland - a methodological exercise," Acta Oncologica, vol. 50, pp. 1235-1243, 2011.

[50] S. H. Sørbye and H. Rue, "Scaling intrinsic gaussian markov random field priors in spatial modelling," Spatial Statistics, vol. 8, pp. 39-51, 2014.

[51] H. Brenner and T. Hakulinen, "Are patients diagnosed with breast cancer before age 50 years ever cured?," Journal of Clinical Oncology, vol. 22, pp. 432438, 2004.

[52] M. A. Luque-Fernandez, A. Belot, M. Quaresma, C. Maringe, M. P. Coleman, and B. Rachet, "Adjusting for overdispersion in piecewise exponential regression models to estimate excess mortality rate in population-based research," BMC Medical Research Methodology, vol. 16, p. 129, 2016.

[53] C. Bauer, J. Wakefield, H. Rue, S. Self, Z. Feng, and Y. Wang, "Bayesian penalized spline models for the analysis of spatio-temporal count data," Statistics in Medicine, vol. 35, pp. 1848-1865, 2016.

[54] Y. Fong, H. Rue, and J. Wakefield, "Bayesian inference for generalized linear mixed models," Biostatistics, vol. 11, pp. 397-412, 2010.

[55] F. Gerber and R. Furrer, "Pitfalls in the implementation of bayesian hierarchical modeling of areal count data: an illustration using bym and leroux models," Journal of Statistical Software, Code Snippets, vol. 63, pp. 1-32, 2015.

\section{Supporting information}

Additional Supporting Information may be found online in the supporting information tab for this article. 


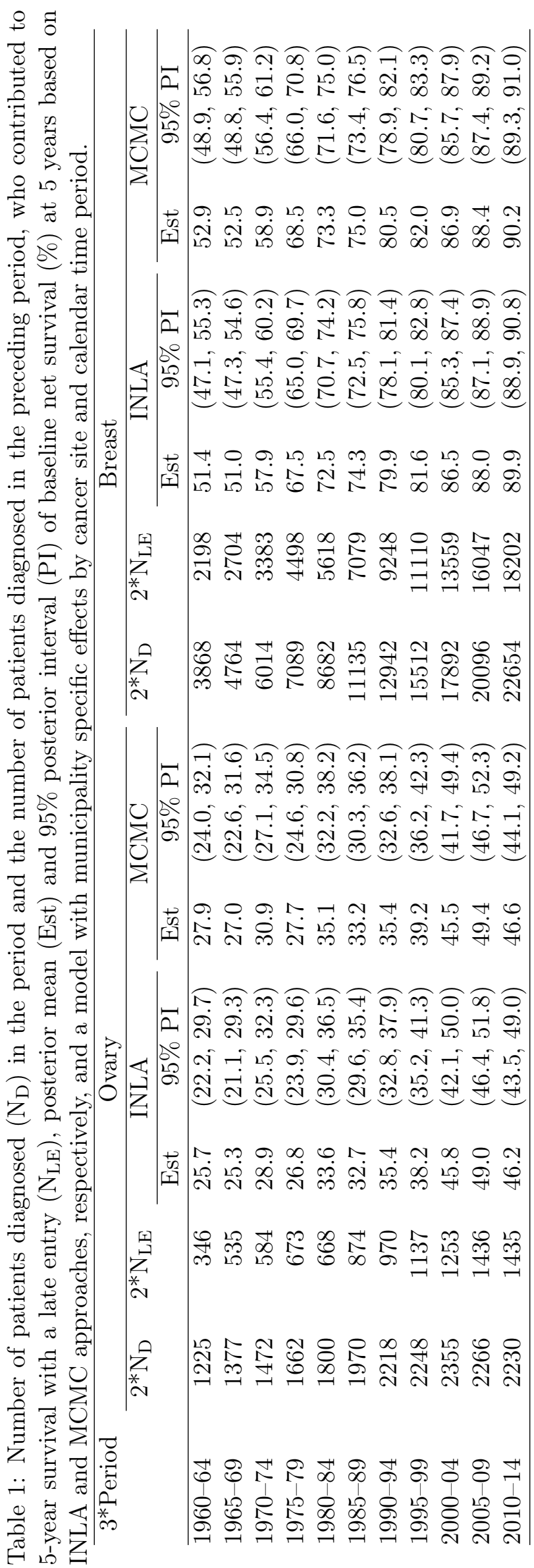



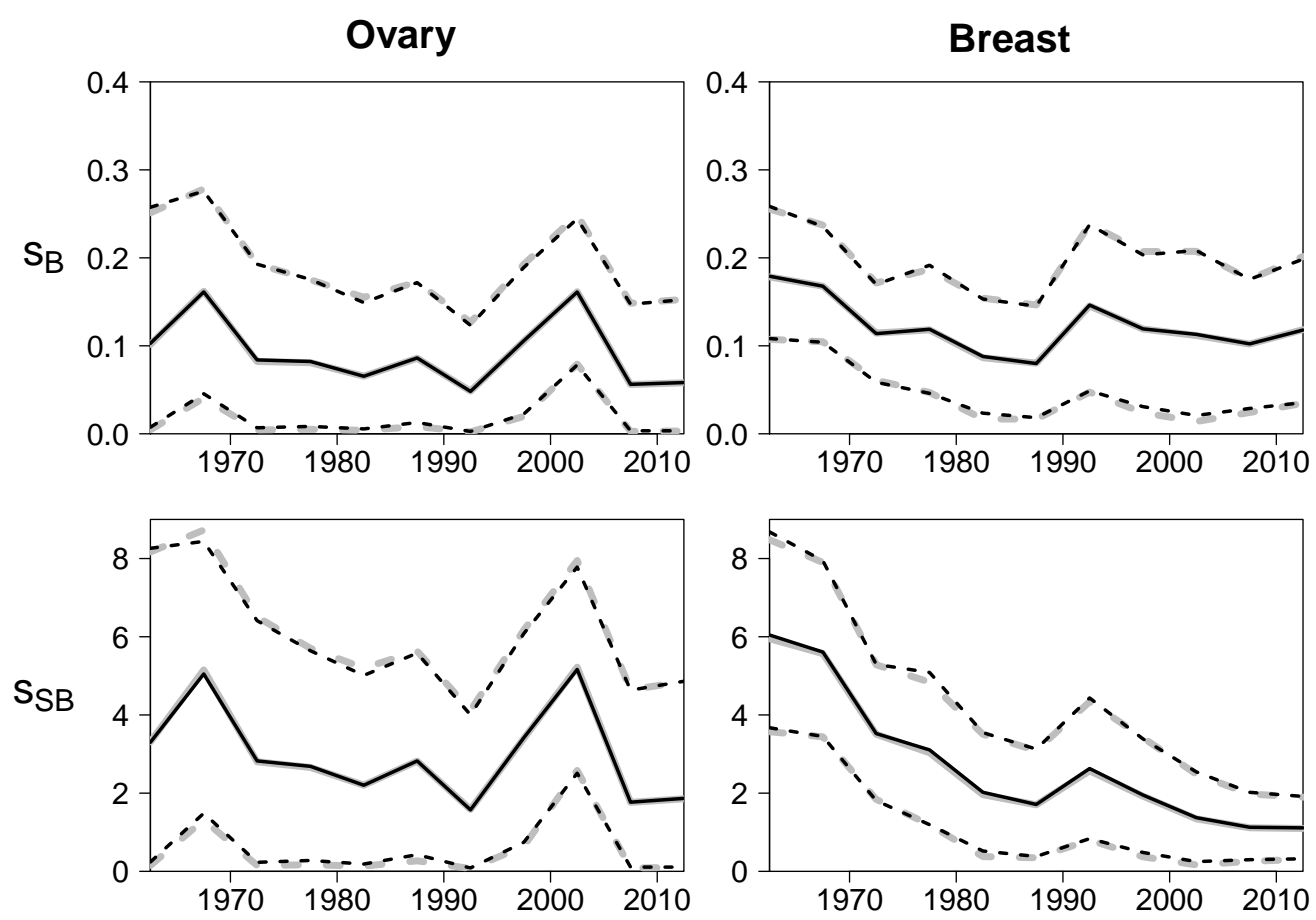

Figure 1: Estimates of INLA (black) and MCMC (gray) based on model (??) without municipality-specific effects: posterior means (solid lines) and 95\% posterior intervals (dashed lines) of the standard deviations $s_{\mathrm{B}}$ and $s_{\mathrm{SB}}$ in female patients diagnosed with cancers of ovary and breast in Finland and followed 5-year periods from 1960-1964 to 2010-2014. 


\section{Ovary}

Breast

Variation between hospital districts
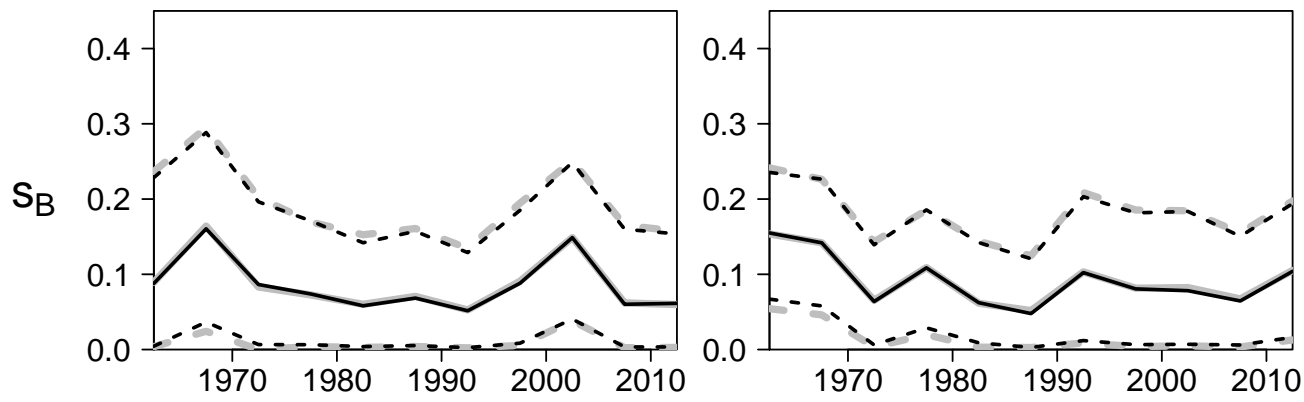

Variation within hospital districts
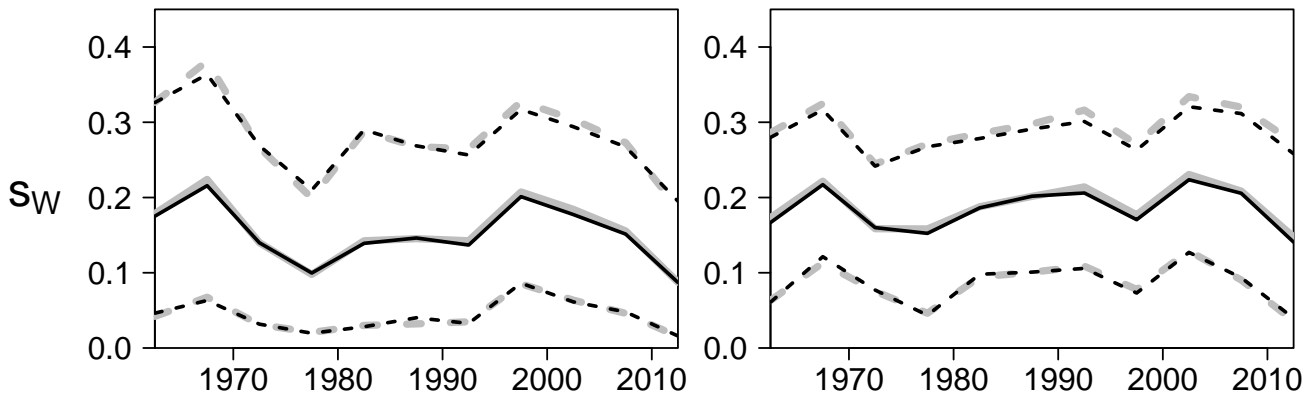

Total variation between municipalities
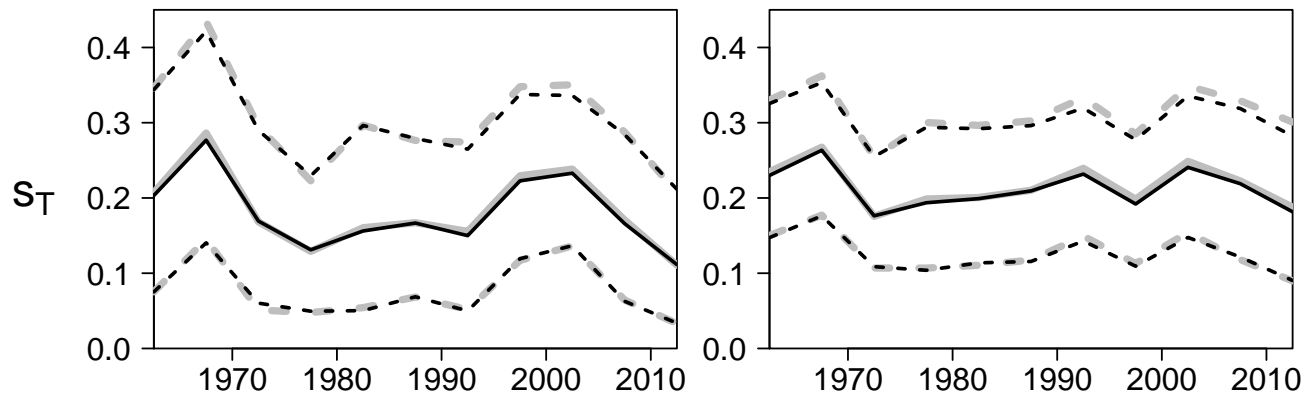

Figure 2: Estimates of INLA (black) and MCMC (gray) based on model (1) that includes both hospital district and municipality specific effects: posterior means (solid lines) and 95\% posterior intervals (dashed lines) of the standard deviations $s_{\mathrm{B}}, s_{\mathrm{W}}$ and $s_{\mathrm{T}}$ in female patients diagnosed with cancers of ovary and breast in Finland and followed in 5-year periods from 1960-1964 to $2010-2014$. 


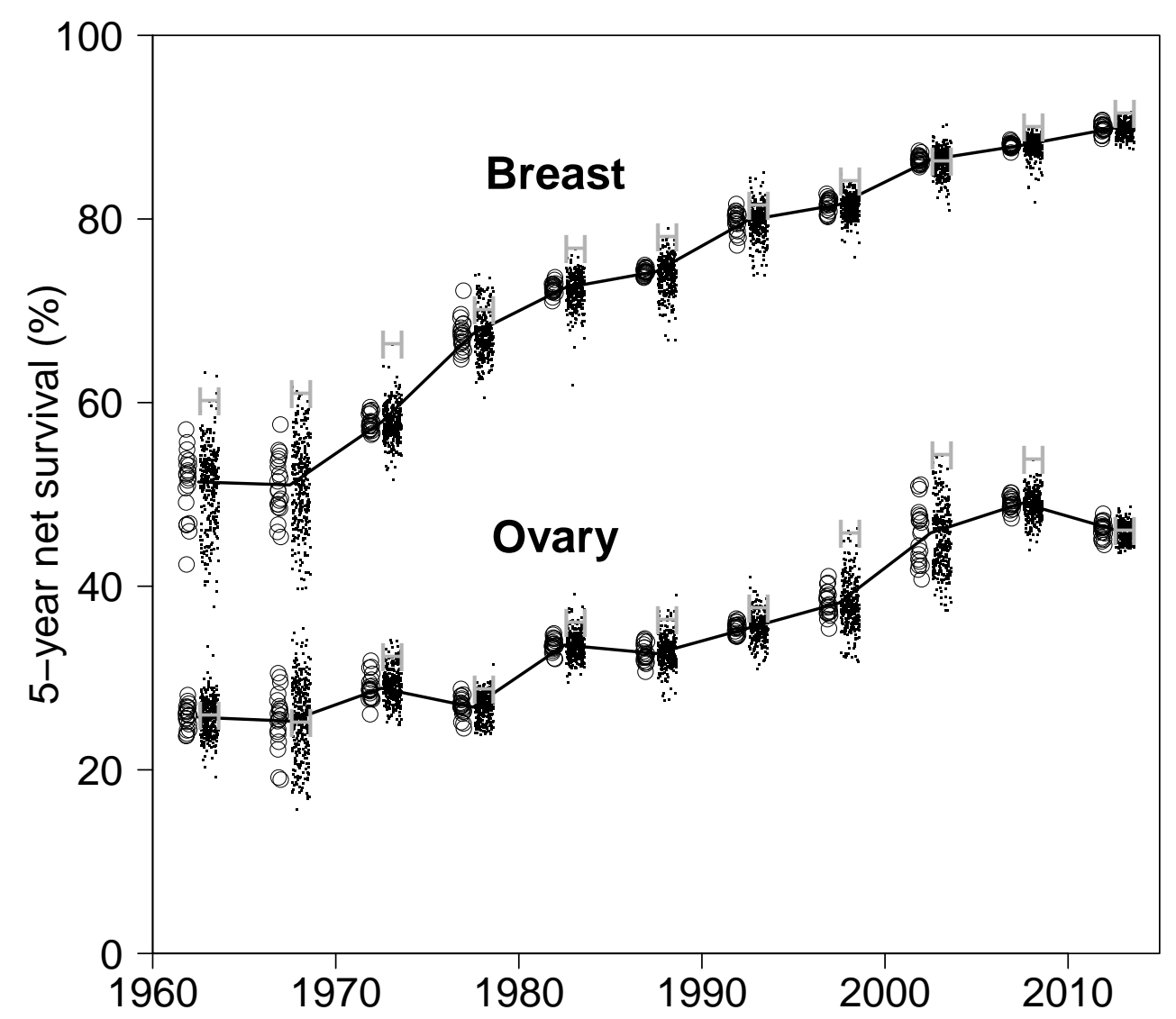

Figure 3: Posterior means of 5-year net survival by hospital districts (open circles) and municipalities (black dots) of patients diagnosed with cancers of ovary and breast in Finland and followed in 5-year periods from 1960-1964 to 2010-2014 based on INLA. Capital letter H shows the estimate of patients diagnosed in the capital of Finland, Helsinki. 
Ovary

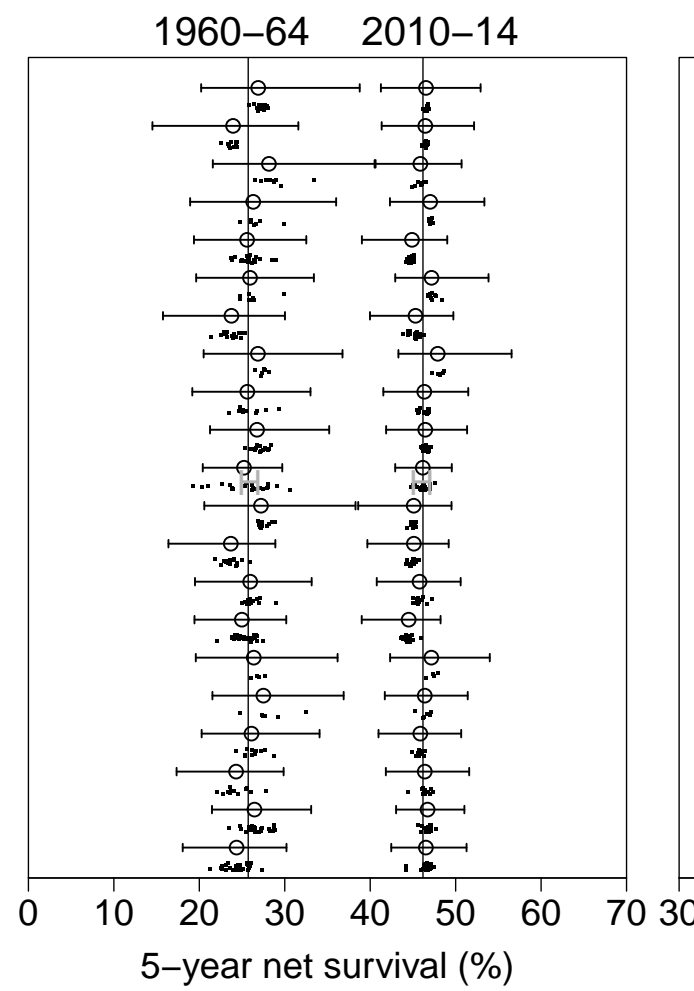

Breast

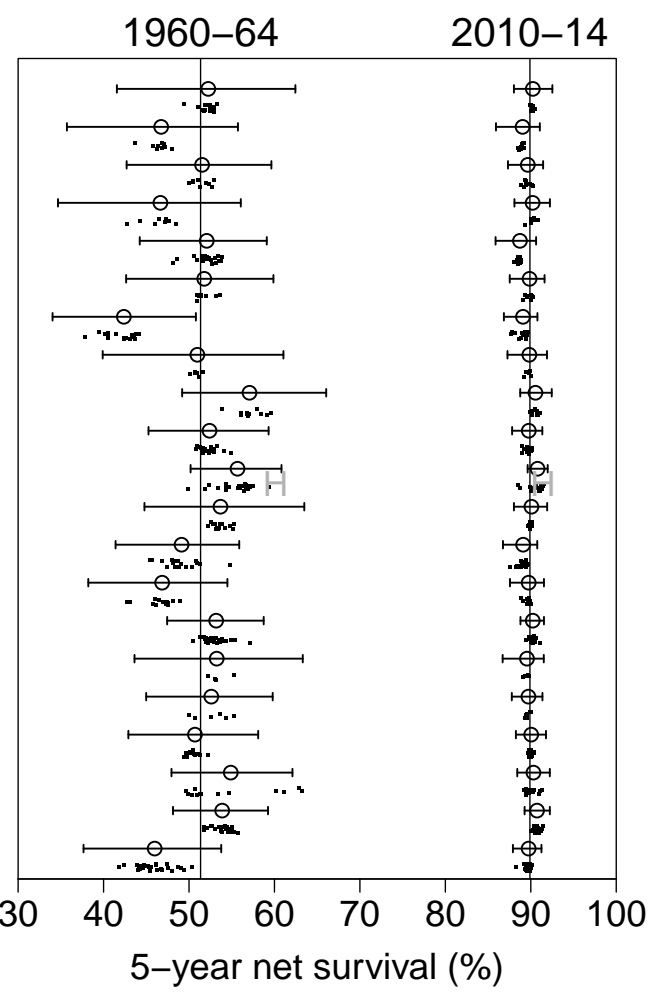

Figure 4: Posterior means of 5-year net survival by hospital districts (open circles) and municipalities (black dots) of patients diagnosed with cancers of ovary and breast in Finland and followed in 1960-1964 and in 20102014 based on INLA. Horizontal lines show $95 \%$ posterior intervals of 5 -year net survival in hospital ditricts, and capital letter $\mathrm{H}$ shows the estimate of patients diagnosed in the capital of Finland, Helsinki. 